\title{
On the frequency selection of finite-amplitude vortex shedding in the cylinder wake
}

\author{
By BENOÎT PIER \\ Department of Applied Mathematics and Theoretical Physics, University of Cambridge, \\ Silver Street, Cambridge CB3 9EW, UK
}

(Received 15 November 2001 and in revised form 26 January 2002)

In this paper it is shown that the two-dimensional time-periodic vortex shedding régime observed in the cylinder wake at moderate Reynolds numbers may be interpreted as a nonlinear global structure and its naturally selected frequency obtained in the framework of hydrodynamic stability theory. The frequency selection criterion is based on the local absolute frequency curve derived from the unperturbed basic flow fields under the assumption of slow streamwise variations. Although the latter assumption is only approximately fulfilled in the vicinity of the obstacle, the theoretically predicted frequency is in good agreement with direct numerical simulations for Reynolds numbers $R e>100$.

\section{Introduction}

Strouhal (1878) appears to be the first to have studied the periodic features produced by the movement of a cylindrical body in air. Ever since, experimental frequency measurements have been refined and the relationship between Strouhal vortex shedding frequency and Reynolds number is now well established, e.g. Provansal, Mathis \& Boyer (1987), Williamson (1988), Norberg (1994), Leweke \& Provansal (1995); for a review see Williamson (1996). On the theoretical side, understanding of the spatiotemporal dynamics of oscillatory flows has proceeded by successively considering linear model equations (Chomaz, Huerre \& Redekopp 1991; Le, Dizès et al. 1996), the linearized version of the Navier-Stokes equations (Monkewitz, Huerre \& Chomaz 1993), and nonlinear model equations on semi-infinite (Couairon \& Chomaz 1996, $1997 a, b, 1999 a, b)$ and infinite (Pier \& Huerre 1996; Pier et al. 1998; Pier, Huerre $\&$ Chomaz 2001) domains. In the framework of the fully nonlinear Navier-Stokes equations, the frequency selection criterion has been obtained (Pier \& Huerre 2001a) for a particular 'synthetic' wake: a wake with no solid obstacle and no reverse flow region. The objective of the present analysis is to demonstrate that the same criterion holds for natural wake flows around solid obstacles.

In the context of spatially developing flows giving rise to self-sustained oscillations, an essential feature is the complex local absolute frequency $\omega_{0}(X)$ (Briggs 1964; Bers 1983; Huerre \& Monkewitz 1990) which depends on the streamwise $X$-coordinate. In absolutely unstable (AU) regions, characterized by $\omega_{0, i}(X) \equiv \operatorname{Im} \omega_{0}(X)>0$, perturbations are not swept away by advection and grow in situ thus leading to non-trivial dynamics without external input. In the past, growing evidence has been gathered to support the existence of a relationship between the global wake frequency and the 
$\omega_{0}(X)$ curve derived from measured or model wake profiles, e.g. Betchov \& Criminale (1966), Koch (1985), Triantafyllou, Triantafyllou \& Chryssostomidis (1986), Monkewitz \& Nguyen (1987), Monkewitz (1988), Hannemann \& Oertel (1989), Karniadakis \& Triantafyllou (1989); for a review see Huerre \& Monkewitz (1990) and Huerre \& Rossi (1998). Different resonance principles have been conjectured: Koch (1985) proposed a feedback mechanism associated with the real absolute frequency $\omega_{0}^{a c} \equiv \omega_{0}\left(X^{a c}\right)$ prevailing at the downstream boundary $X^{a c}$ of the AU region. Monkewitz \& Nguyen (1987) considered an initial resonance principle where the real global frequency $\omega_{0}^{c a} \equiv \omega_{0}\left(X^{c a}\right)$ is provided by the upstream transition station $X^{c a}$ from convective to absolute instability.

According to the first theoretically consistent criterion established in a strictly linear setting by Chomaz et al. (1991), Monkewitz et al. (1993) and Le Dizès et al. (1996), the complex global frequency $\omega_{s}^{\ell}$ is given by a saddle-point condition

$$
\omega_{s}^{\ell}=\omega_{0}\left(X_{s}^{\ell}\right) \quad \text { with } \quad \frac{\mathrm{d} \omega_{0}}{\mathrm{~d} X}\left(X_{s}^{\ell}\right)=0,
$$

based on the analytic continuation of $\omega_{0}(X)$ in the complex $X$-plane. This linear criterion was shown by Hammond \& Redekopp (1997) to yield a strikingly accurate frequency prediction for the fully developed vortex street in the wake of a bluntedged plate. Note, however, that $\omega_{0}(X)$ in that study is based on the time-averaged mean flow instead of the unperturbed basic flow, thus implicitly taking into account nonlinear effects. The performance of criterion (1.1) based on mean flows will be discussed in the final section.

In the framework of fully nonlinear amplitude evolution equations and by investigating semi-infinite domains, Couairon \& Chomaz (1997a, b, 1999a, b) have derived scaling laws that are in excellent agreement with experimental and numerical results pertaining to the spatial structure of bluff-body wakes close to threshold. In infinite systems, self-sustained time-periodic finite-amplitude structures have been found as soft ('hat') modes (Pier \& Huerre 1996) or steep ('elephant' $\dagger$ ') modes (Pier et al. 1998), and the respective frequency selection criteria have been established. The analysis of the relevant transition scenarios (Pier et al. 2001) has shown that the unperturbed basic state always first bifurcates to an elephant structure. Moreover, hat modes may only exist in situations of weak mean flow advection, so that they are ruled out in wake flows. Nonlinear elephant modes are characterized by a stationary front located at the upstream transition station $X^{c a}$ from local convective to absolute instability. This front acts as a source generating a downstream-propagating nonlinear wavetrain and effectively tuning the entire system to its frequency. The stationary front obeys the Dee \& Langer (1983) marginal stability criterion, hence the frequency of elephant modes equals the real absolute frequency prevailing at the front location and is given by

$$
\omega_{0}^{c a}=\omega_{0}\left(X^{c a}\right) \quad \text { with } \quad \omega_{0, i}\left(X^{c a}\right)=0 \quad \text { and } \quad \frac{\mathrm{d} \omega_{0, i}}{\mathrm{~d} X}\left(X^{c a}\right)>0 .
$$

Downstream of the front, a fully nonlinear wavetrain prevails that is governed by the local nonlinear dispersion relation and follows the nonlinear wavenumber branch of frequency $\omega_{0}^{c a}$. It should be noted that this criterion governing a fully nonlinear global structure only involves the purely linear local absolute frequency, and that it fully confirms the conjecture of Monkewitz \& Nguyen (1987).

All theoretical analyses rely on slow streamwise variations to establish the relationship between global behaviour and local characteristics. In this context, the linear (1.1)

$\dagger$ The choice of the terms 'hat' and 'elephant' is motivated by Pier \& Huerre (2001b). 
and nonlinear (1.2) criteria yield a leading-order approximation of the respective global frequency, and corrections of higher order in the inhomogeneity parameter are obtained by further asymptotic analyses (Monkewitz et al. 1993; Pier et al. 2001). In order to solve the global mode problem in the context of the fully nonlinear NavierStokes equations, a 'synthetic' wake (Pier \& Huerre 2001a) was designed that fulfils the quasi-parallel flow assumption. In this configuration, local linear and nonlinear dispersion relations derived from the basic flow velocity profiles predict the existence of a nonlinear elephant mode, the global frequency and spatial structure of which are in excellent agreement with the synchronized finite-amplitude vortex street obtained by direct numerical simulations.

Whereas the synthetic wake was tailored to obey the assumption of slow streamwise variations required by theory, bluff body wakes are strongly non-parallel near the obstacle surface. Nevertheless, the present study has been undertaken, bearing in mind that perturbation analyses often yield reasonable predictions for finite values of the 'small' parameter. The results then validate a posteriori the method and assess the utility of asymptotic analyses of quasi-parallel flows in situations of practical interest.

\section{Governing equations and solution method}

The following study is carried out for two-dimensional incompressible flows governed by the Navier-Stokes equations. The Reynolds number is defined as $R e=U D / v$, where $U$ represents the free-stream velocity, $D$ the obstacle diameter and $v$ the kinematic viscosity. Using non-dimensional variables based on $D$ and $U$, the governing momentum and continuity equations are then given by

$$
\begin{aligned}
\frac{\partial u}{\partial t}+u \frac{\partial u}{\partial x}+v \frac{\partial u}{\partial y} & =-\frac{\partial p}{\partial x}+\frac{1}{R e} \Delta u+f_{u} \\
\frac{\partial v}{\partial t}+u \frac{\partial v}{\partial x}+v \frac{\partial v}{\partial y} & =-\frac{\partial p}{\partial y}+\frac{1}{R e} \Delta v+f_{v} \\
\frac{\partial u}{\partial x}+\frac{\partial v}{\partial y} & =0
\end{aligned}
$$

where $x$ and $y$ denote streamwise and cross-stream coordinates, $u$ and $v$ the corresponding components of the velocity and $p$ the pressure field.

For fast numerical integration, the above equations are discretized on a Cartesian grid, and the presence of the obstacle is enforced by a penalization method similar to that used by Angot, Bruneau \& Fabrie (1999): inside the domain covered by the cylinder $\left(x^{2}+y^{2} \leqslant 1 / 4\right)$, a 'body force' is applied with components

$$
f_{u}=-u / \tau \text { and } f_{v}=-v / \tau \text {, }
$$

where $\tau$ is a relaxation parameter. A value of $\tau=0.01$ was found sufficient to drive the components of the total velocity field to negligible values, and results are not affected when further decreasing $\tau$. Thus, the entire domain is filled with fluid and there is no need for body-fitted coordinates or for boundary conditions on the obstacle surface.

Spatial discretization combines finite differences with $n_{x}=n_{u}+n_{o}+n_{d}$ points in the $x$-direction and $n_{y}$ Chebyshev collocation points in the $y$-direction. The streamwise mesh is constructed with $n_{o}$ equispaced grid points separated by $\delta_{x}$ in the obstacle region and $n_{u}\left(n_{d}\right)$ elements in the upstream (downstream) regions uniformly stretched according to a stretching factor of $\kappa_{u}\left(\kappa_{d}\right)$. The Chebyshev collocation points $-1 \leqslant$ 


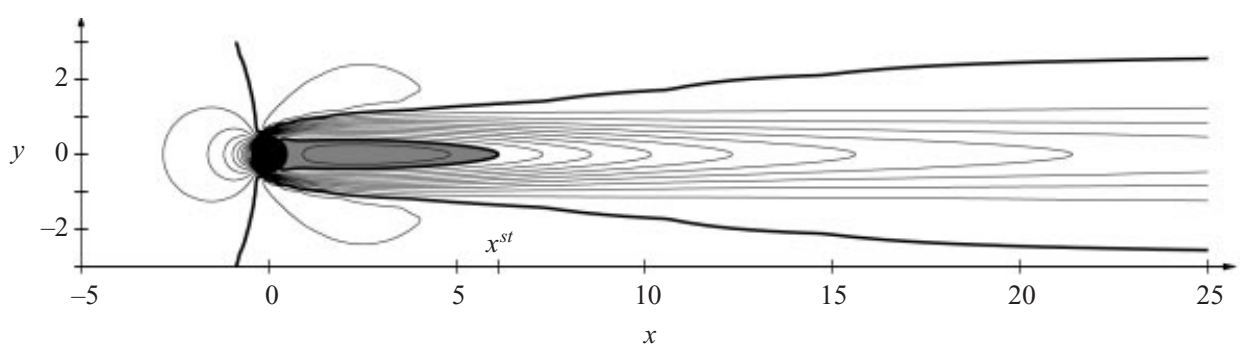

FIGURE 1. Streamwise velocity field of the basic flow around the cylinder at $R e=100$. Isolines pertain to the levels $-0.1,0.0, \ldots, 1.1(0,1$ thick lines). A reverse flow region $(u<0$, shown in grey) prevails between the obstacle and the stagnation point at $x^{\text {st }}$.

$\xi_{i}=-\cos \left[i \pi /\left(n_{y}+1\right)\right] \leqslant+1$ for $i=0, \ldots, n_{y}+1$ are mapped onto the entire crossstream axis $-\infty \leqslant y_{i} \leqslant+\infty$ through the algebraic transformation $y \sqrt{2} / \ell_{y}=\xi /\left(1-\xi^{2}\right)$, where the parameter $\ell_{y}$ governs the distribution of collocation points on the $y$-axis. Assuming that $u-U, v$ and $p$ vanish at $y= \pm \infty$, the computation may be restricted to the interior collocation points associated with $1 \leqslant i \leqslant n_{y}$. At the inlet the freestream velocity is imposed, and at the outlet non-reflecting boundary conditions (Jin $\&$ Braza 1993) are implemented. The grid used in the present computations is defined by $n_{y}=55, \ell_{y}=1$ and $n_{u}=60, n_{o}=140, n_{d}=300, \delta_{x}=0.01, \kappa_{u}=1.10, \kappa_{d}=1.02$; the total streamwise extent of the domain is then $-35<x<195$.

Time-integration of system (2.1) is performed via a fractional-step method of second-order accuracy in time. At the intermediate time-step, the two components of the velocity field are obtained by solving Helmholtz-type problems. A Poisson problem then yields a correction to the pressure required to enforce divergence-free velocity fields. A Crank-Nicholson scheme is used for the viscous terms; the advection terms are obtained at the intermediate time-step by extrapolation based on the two previous time-steps.

Using a Cartesian grid, the second-order $y$-derivative operator may be diagonalized so that the two-dimensional Helmholtz (Poisson) problems transform into a series of decoupled one-dimensional problems $\uparrow$ that are efficiently solved by making use of a generalized Thomas algorithm. Thus the required computational time only increases linearly with the total number of grid points. Most of the results have been obtained on a laptop computer; a typical run takes on the order of one hour.

\section{Basic flow and local absolute frequency}

This part of the study investigates the unperturbed basic wake flow and the corresponding local absolute frequency curve, upon which the frequency selection criteria are based. By definition, the basic flow is a steady solution of the NavierStokes equations (2.1). This flow is unstable for Reynolds numbers beyond critical and then impossible to observe experimentally. Following Fornberg (1985), however, the steady solution is obtained when imposing a stabilizing symmetry condition on the $y=0$ axis and considering only the domain $y \geqslant 0$. Figure 1 shows the basic streamwise velocity field around the circular cylinder at $R e=100$. The near wake displays a reverse flow region $(u<0$, shown in grey) extending from the obstacle

$\dagger$ The author is indebted to Uwe Ehrenstein for bringing this method to his attention. 

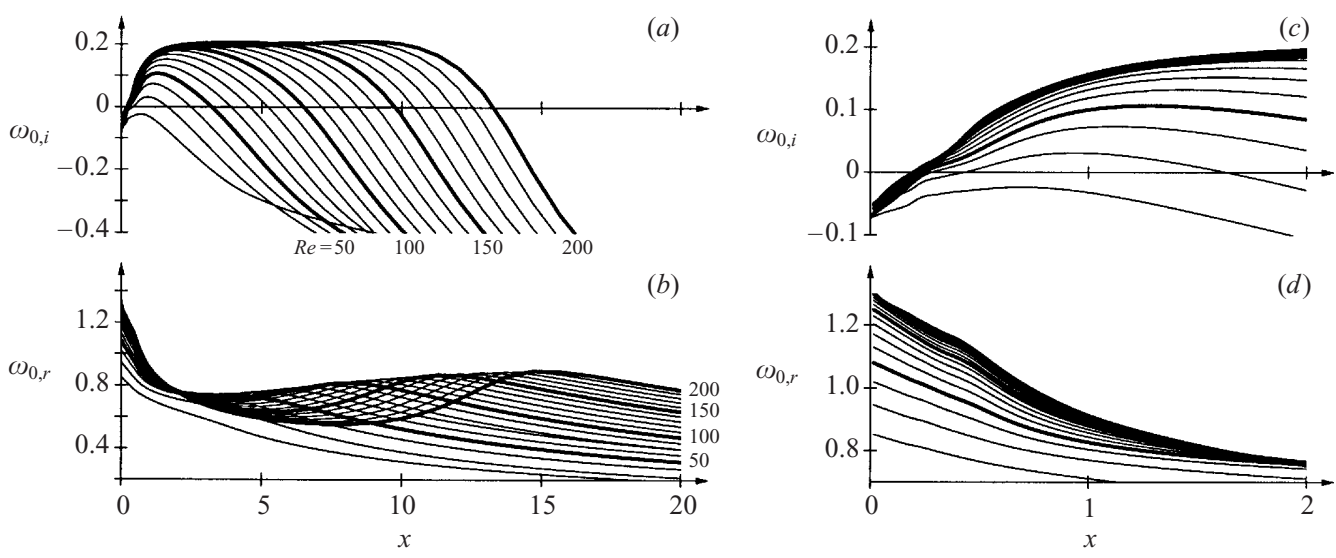

FiguRE 2. Imaginary $(a, c)$ and real $(b, d)$ parts of local absolute frequency for basic wake flows obtained with $R e=20,30, \ldots, 200$ (thick curves pertain to $R e=50,100,150,200$ ).

down to the stagnation point $x^{s t}$. The variation with Reynolds number of the reverse flow extent is discussed below (see figure 3 ).

In a previous investigation (Pier \& Huerre 2001a) resorting to the academic configuration of a 'synthetic' wake with no solid boundaries, no reverse flow and no stagnation point, the existence of a small inhomogeneity parameter $\epsilon \ll 1$ made possible a rigorous asymptotic analysis based on the separation of fast $x$ and slow $X \equiv \epsilon x$ streamwise scales. In an attempt to prove the applicability of these results in situations of practical interest where $\epsilon=O(1)$, we boldly ignore that the present flow is non-parallel in the near-wake region and do not use the slow $X$-coordinate in the rest of the paper. Local characteristics are then derived at a given streamwise station by freezing the $x$-coordinate and studying the equivalent parallel shear flow of velocity profile $U_{0}(y)=u(x, y)$. Linear instability waves are governed by the Orr-Sommerfeld equation (Drazin \& Reid 1981) which yields the local linear dispersion relation $\omega=\Omega^{\ell}(k, x)$ between the complex frequency $\omega$ and complex wavenumber $k$ at the streamwise station $x$ under consideration. These linear eigenvalue problems in the cross-stream coordinate are solved via a Chebyshev spectral method based on the previously introduced collocation points. The complex local absolute frequency $\omega_{0}(x)$ is then derived in classical fashion by applying a zero group velocity condition on the local linear dispersion relation (Briggs 1964; Bers 1983). Figure 2 illustrates the streamwise evolution of the absolute growth rate $\omega_{0, i}$ and real absolute frequency $\omega_{0, r}$ for different values of the Reynolds number in the range $20 \leqslant R e \leqslant 200$. Local absolute instability $\left(\omega_{0, i}>0\right)$ prevails downstream of the obstacle when $R e>R e_{a} \simeq 25$, in agreement with the findings of Monkewitz (1988). The magnified graphs (figure $2 c, d$ ) show that the near wake is insensitive to changes in $R e$ when $R e>100$. It should also be noted that the local absolute growth rate rapidly decays with decreasing $x$ in the boundary layers along the cylinder $(-0.5<x<0.5)$ and reaches very large negative values for $x<-0.5$ (not shown on graph).

The evolution with Reynolds number of the AU and reverse flow regions is shown in figure 3. Absolute instability prevails in the interval $x^{c a}<x<x^{a c}$ (both shades of grey), the extent of which is seen to increase approximately linearly with $R e$. Note that the maximum absolute growth rate remains almost constant for $R e>100$ (see figure $2 a$ ). A comparison of the stagnation point location $x^{\text {st }}$ (thick solid curve 


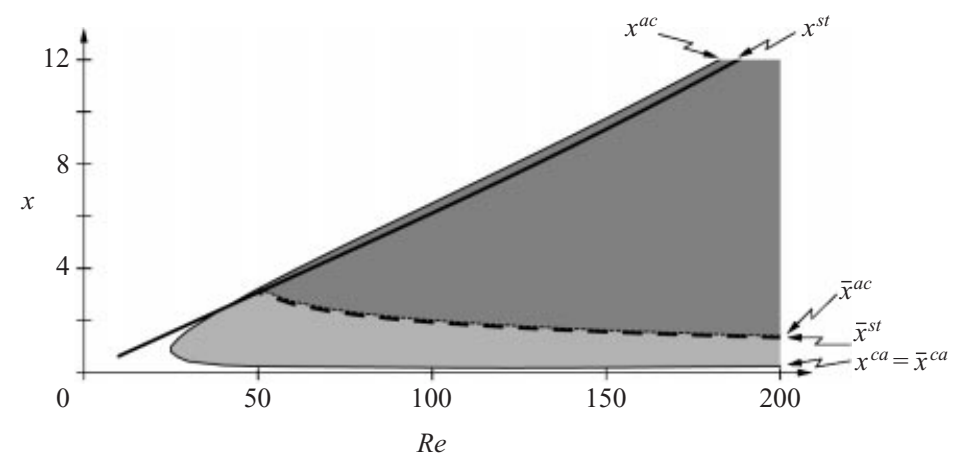

FIGURE 3. Reynolds number dependence of local absolute instability and reverse flow region for the basic flow (AU region extends over both shaded areas $x^{c a}<x<x^{a c}$ and the stagnation point location $x^{\text {st }}$ follows the thick solid curve) and the mean flow (AU region covers light grey area $\bar{x}^{c a}<x<\bar{x}^{a c}$ and stagnation point $\bar{x}^{s t}$ follows the thick dashed curve).

in figure 3) and the marginally absolutely unstable station $x^{a c}$ shows that the AU domain closely follows the reverse flow region, slightly extending beyond it. The remaining elements of figure 3 pertain to the time-averaged mean flows and are discussed below.

\section{Periodic vortex shedding}

Above a critical value of Reynolds number, finite-amplitude periodic vortex streets develop in the wake of the cylinder when the entire cross-stream domain $-\infty \leqslant y \leqslant$ $+\infty$ is considered. Direct numerical simulations of system (2.1) are performed using the method outlined in $\S 2$. Simulations are started with the basic flow fields and the instability is triggered by a small-amplitude initial impulse. After a transient growth, nonlinearities rapidly lead to saturation of a fully developed downstream-propagating periodic vortex street. Figure 4 shows a snapshot of the velocity fields at $R e=100$ in the central region of the computational domain. Time series are recorded at different locations and corresponding frequency spectra computed (figure 5) to ascertain the synchronized behaviour of the flow. Inspection of these spectra demonstrates that the entire vortex street is tuned to a global fundamental frequency and its harmonics. Figure 6 shows that the numerically obtained frequencies (open squares) are in good agreement with the experimental relationship (solid curve) between Strouhal number $S t=\omega / 2 \pi$ and Reynolds number (Williamson 1988). Moreover, the critical Reynolds number $R e_{c}$ for onset of periodic vortex shedding has been localized in the range $49.0<R e_{c}<49.5$ which nearly corresponds to the experimental threshold of 47 measured by Provansal et al. (1987). Bearing in mind that the cylindrical obstacle is discretized on a Cartesian grid, these agreements are deemed sufficient validation of the code for the present purpose; more accurate numerical results have been obtained e.g. by Barkley \& Henderson (1996).

The nonlinear vortex street is associated with Reynolds stresses leading to a finite mean flow correction. The main effect of this mean flow distortion is a shortening of the recirculation bubble, as indicated by the stagnation point location $\bar{x}^{\text {st }}$ of the time-averaged flows (thick dashed curve in figure 3). It should be noted that the average flow is a result of the nonlinear vortex street and does not obey the Navier-Stokes equations. For comparison, however, the local linear stability analysis of the previous section may be repeated for the time-averaged flows. This reveals that 

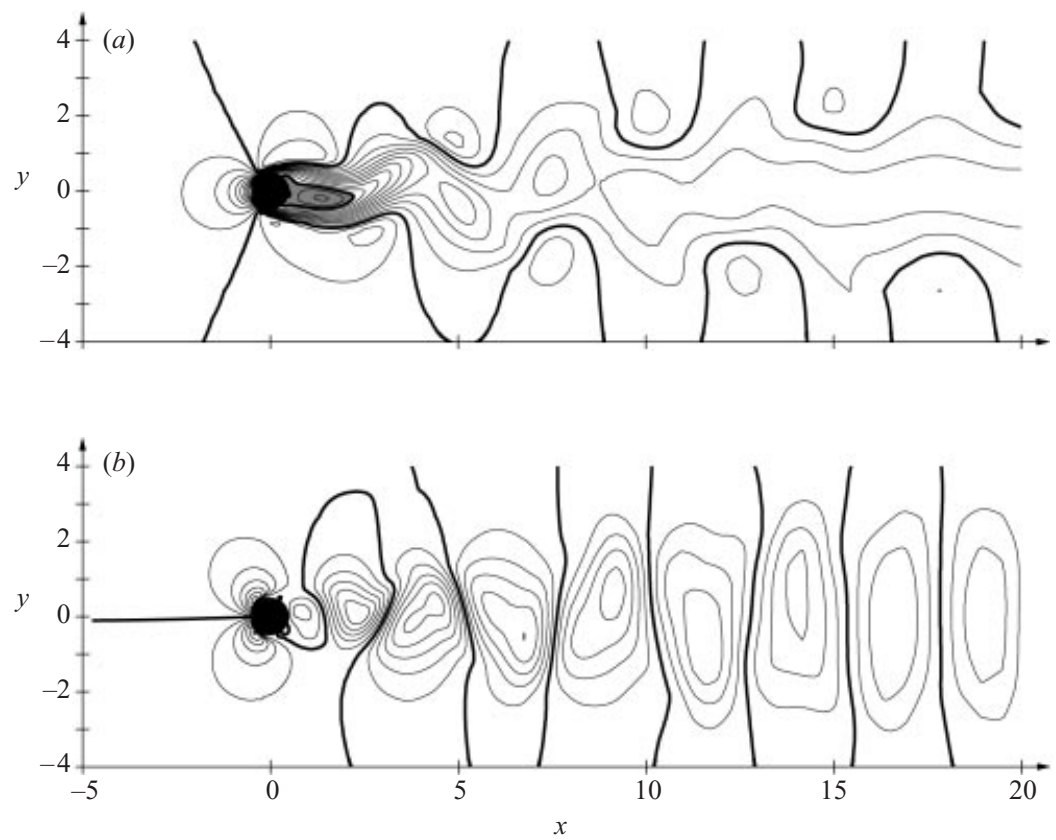

FIGURE 4. Snapshot of $(a)$ streamwise and $(b)$ cross-stream velocity fields in the periodic nonlinear vortex shedding régime at $R e=100$. (a) Contour levels $u=-0.2,-0.1, \ldots, 1.2(0,1$ thick lines). (b) Contour levels $v=-0.6,-0.5, \ldots, 0.6$ (0 thick line).
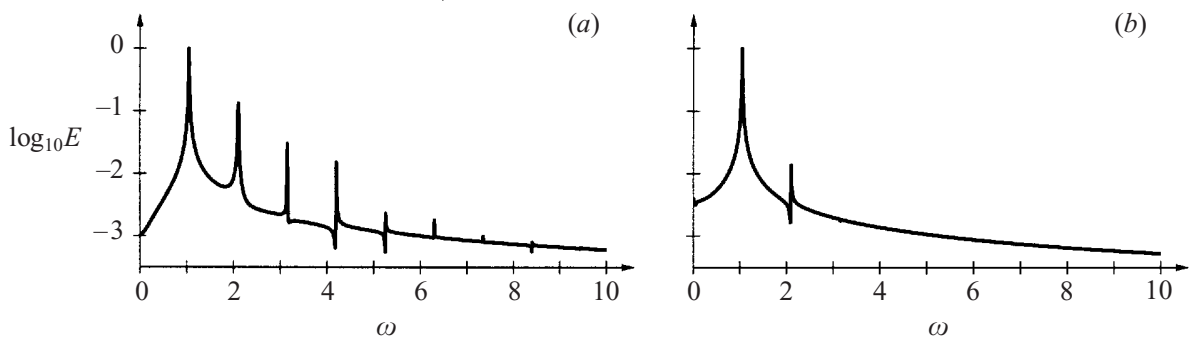

FiguRE 5. Harmonic spectra of velocity time series in the vortex shedding régime at $R e=100$. (a) $v$-component at $x=2.2, y=0.7$. (b) $u$-component at $x=0.2, y=2.1$.

the reduction of the reverse flow region is associated with a similar reduction of the absolutely unstable domain $\left(\bar{x}^{c a}<x<\bar{x}^{a c}\right.$, lightly shaded region in figure 3$)$. There is, however, no feedback of the nonlinear downstream vortices in the upstream region; in particular the neighbourhood of the upstream boundary $x^{c a}=\bar{x}^{c a}$ of the AU region is not affected by mean flow corrections.

\section{Discussion of frequency selection criterion}

The objective of this last section is to show that the vortex shedding régime may be interpreted in terms of a nonlinear elephant mode, i.e. that its global frequency is dictated by criterion (1.2). The frequency of nonlinear elephant global modes is imposed by a sharp front located at the transition station $x^{c a}$ from local convective to absolute instability. The global frequency of the system then equals the real absolute 


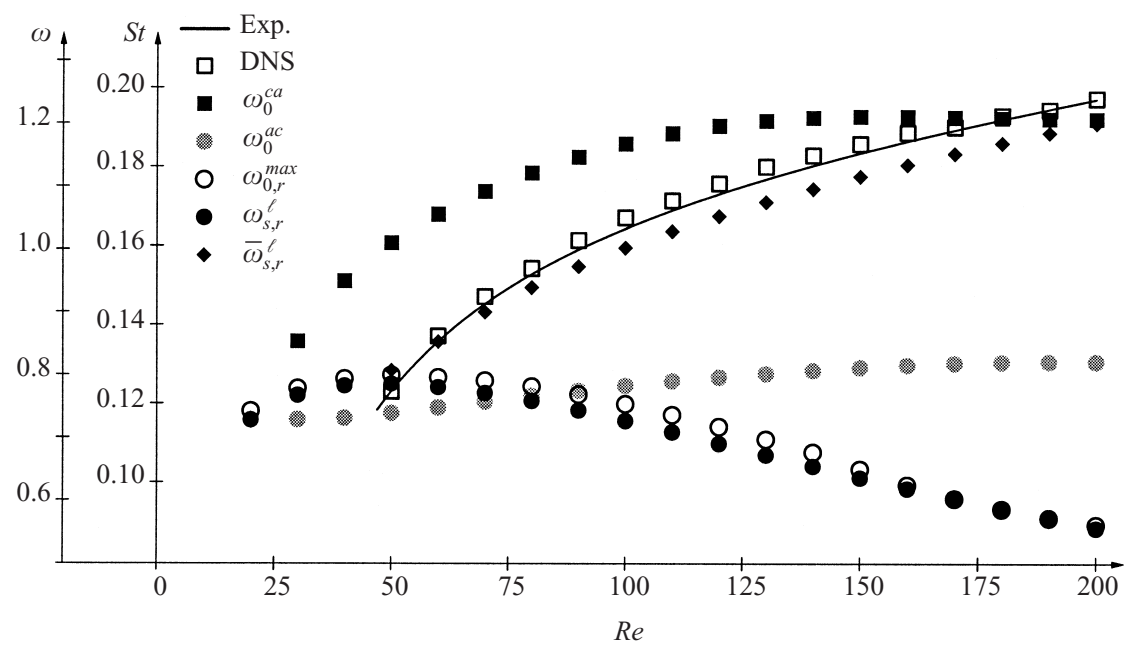

FIGURE 6. Reynolds number dependence of cylinder wake characteristic frequencies. Vortex shedding frequencies of the present simulations (open squares) closely follow the experimental Strouhal number curve from Williamson (1988) (solid line). Theoretical elephant frequencies $\omega_{0}^{c a}$ (filled squares) approximately predict the actual vortex shedding frequencies for $R e>100$. The other characteristic frequencies $\omega_{0}^{a c}$ (grey circles), $\omega_{0, r}^{\max }$ (open circles) and $\omega_{s, r}^{\ell}$ (filled circles) are unable to account for the fully developed vortex street beyond onset at $R e \simeq 49$. Note the good performance of $\bar{\omega}_{s, r}^{\ell}$ based on the mean flow (diamonds).

frequency $\omega_{0}^{c a}$ prevailing at $x^{c a}$. For the wake flows under consideration, transition from convective to absolute instability occurs in the boundary layer along the cylinder near its trailing edge, as demonstrated by figure 2(c). Monitoring the corresponding frequency $\omega_{0}^{c a}$ as the Reynolds number is varied (filled squares in figure 6) shows that this frequency plateaus at $\omega_{0}^{c a} \simeq 1.2$ for $R e>100$. Comparison of these theoretical predictions with the results obtained by the present simulations (open squares) or by experiment (solid line) shows that the discrepancy is less than $10 \%$ over the entire range $100<R e<200$. Thus the theory is fairly successful in predicting the actual vortex shedding frequency, considering that criterion (1.2) is a leading-order approximation derived under the condition of slow streamwise evolution of the entire flow. The agreement improves at higher Reynolds numbers since then the assumption of weakly diverging flows is more closely fulfilled. Note also that perfect agreement occurs at $R e=180$, beyond which the two-dimensional vortex street undergoes a transition to three-dimensionality (Barkley \& Henderson 1996; Williamson 1996). For $R e<100$, finite non-parallel effects result in a poorer frequency prediction. This strong non-parallelism may also account for the discrepancy between onset of absolute instability at $R e=R e_{a} \simeq 25$ and onset of global instability at $R e=R e_{c} \simeq 47$. Indeed, in the range $R e_{a}<R e<R e_{c}$ the extent of the AU region is much less than the typical vortex street wavelength and thus unable to sustain a stationary front.

The mechanism leading to the synchronized vortex street at moderate Reynolds numbers may be interpreted in the following way. The initial impulse triggers a wavepacket of growing amplitude and its envelope advances against the basic flow in the absolutely unstable region. Perturbations thus penetrate into the boundary layer near the cylinder trailing edge. Further upstream, at the station $x^{c a}$ of neutral 
absolute instability, a balance between upstream perturbation growth and downstream advection is reached, and perturbations pile up at that location. Nonlinearities lead to saturation of the fluctuating amplitude, a front is formed at $x^{c a}$ and a fully nonlinear wavetrain obtained in the region $x>x^{c a}$. Its frequency is dictated by the front and is $\omega_{0}^{c a}$. The domain $x<x^{c a}$ is covered by the front tail and the fluctuation amplitude exponentially decays towards the inlet. At the marginal $x^{c a}$ station, weakly nonlinear fluctuations prevail (as illustrated in figure $5(b)$ for $R e=100$ when $x^{c a}=0.2$ ). It should be emphasized that perturbations evolve in the infinite $-\infty<x<+\infty$ system and no boundary condition is imposed at the obstacle trailing edge $x=0.5$. This is in contrast with the investigations of semi-infinite domains with upstream boundary condition by Couairon \& Chomaz $(1997 a, b, 1999 a, b)$. In the latter configuration, analysis of one-dimensional model equations close to global instability yields scaling laws that account for experimental observations remarkably well. The present study does not rely on the assumption of near criticality, but rather on reasonably parallel flows as obtained for $R e>100$.

To fully appreciate the quality of criterion (1.2) in predicting the vortex shedding frequency, it should be compared with the other basic flow characteristic frequencies (round symbols on figure 6). The filled grey circles represent the real absolute frequency $\omega_{0}^{a c}$ prevailing at the downstream boundary $x^{a c}$ of the AU region. Although the location $x^{a c}$ continuously moves downstream with increasing Reynolds number (see figure $3 a$ ), the frequency $\omega_{0}^{a c}$ is seen to remain approximately constant at 0.8 . Another characteristic frequency is derived from the location $x^{\max }$ where the absolute frequency $\omega_{0}^{\max } \equiv \omega_{0}\left(x^{\max }\right)$ with maximum absolute growth rate occurs. Its real part $\omega_{0, r}^{\max }$ is plotted by open circles in figure 6. The filled circles in figure 6 represent $\omega_{s, r}^{\ell} \equiv \operatorname{Re} \omega_{s}^{\ell}$ of criterion (1.1) governing spatially extended and globally synchronized structures when the governing equations are linearized with respect to the basic flow. The frequencies $\omega_{s}^{\ell}$, obtained by analytic continuation of the $\omega_{0}(x)$ curves in the complex $x$-plane, are found to approximately follow $\omega_{0}^{\max }$ since the complex saddle point $x_{s}^{\ell}$ is located near the position $x^{\max }$ of maximum absolute growth rate. Both $\omega_{0, r}^{\max }$ and $\omega_{s, r}^{\ell}$ display even lower values than $\omega_{0}^{a c}$ for $R e>100$ and none of these frequencies can account for the actual global frequency. This is not a surprise since they are not derived from a nonlinear theory. In contrast, as already established by Hammond \& Redekopp (1997) in a similar configuration, the linear criterion (1.1) applied on the mean flows obtained by averaging in time the fully nonlinear régime yields frequencies $\bar{\omega}_{s, r}^{\ell}$ (diamonds in figure 6) which very closely follow the actually observed frequencies. Unfortunately, since these frequencies are based on the fully developed vortex street, this excellent agreement does not help identifying the mechanism that is responsible for the vortex shedding.

To conclude, it has been shown that the vortex shedding frequency governing bluff body wakes can be understood and predicted by local instability analyses of the basic flow considered to be weakly diverging. Despite non-parallel effects in the vicinity of the obstacle, reasonable agreement is obtained for $R e>100$. It should also be emphasized that the aim of the present method is to reveal the underlying frequency selection mechanism and not to accurately estimate the global frequency: the frequency predictions require almost the same numerical effort as the complete simulations!

Present and past fruitful collaborations with Nigel Peake and Patrick Huerre, suggestions and advice from Uwe Ehrenstein, John Hinch, Paul Metcalfe and Laurette Tuckerman are gratefully acknowledged. 


\section{REFERENCES}

Angot, P., Bruneau, C.-H. \& Fabrie, P. 1999 A penalization method to take into account obstacles in incompressible viscous flows. Numer. Maths 81, 497-520.

BARKLey, D. \& Henderson, R. 1996 Three-dimensional Floquet stability analysis of the wake of a circular cylinder. J. Fluid Mech. 322, 215-241.

BERs, A. 1983 Space-time evolution of plasma instabilities - absolute and convective. In Handbook of Plasma Physics (ed. M. Rosenbluth \& R. Sagdeev), pp. 451-517. North-Holland.

Betchov, R. \& Criminale, W. 1966 Spatial instability of the inviscid jet and wake. Phys. Fluids $\mathbf{9}$, 359-362.

BRIGGS, R. 1964 Electron-Stream Interaction with Plasmas. M.I.T. Press.

Chomaz, J.-M., Huerre, P. \& Redekopp, L. 1991 A frequency selection criterion in spatially developing flows. Stud. Appl. Maths 84, 119-144.

Couarron, A. \& Chomaz, J.-M. 1996 Global instabilities in fully nonlinear systems. Phys. Rev. Lett. 77, 4015-4018.

Couairon, A. \& Chomaz, J.-M. 1997 a Absolute and convective instabilities, front velocities and global modes in nonlinear systems. Physica D 108, 236-276.

Couniron, A. \& Chomaz, J.-M. $1997 \mathrm{~b}$ Pattern selection in the presence of a cross flow. Phys. Rev. Lett. 79, 2666-2669.

Couniron, A. \& Chomaz, J.-M. 1999a Fully nonlinear global modes in slowly varying flows. Phys. Fluids 11, 3688-3703.

Couniron, A. \& Chomaz, J.-M. $1999 b$ Primary and secondary nonlinear global instability. Physica D 132, 428-456.

Dee, G. \& Langer, J. 1983 Propagating pattern selection. Phys. Rev. Lett. 50, 383-386.

Drazin, P. \& ReID, W. 1981 Hydrodynamic Stability. Cambridge University Press.

FORNBERG, B. 1985 Steady viscous flow past a circular cylinder up to Reynolds number 600 J. Comput. Phys. 61, 297-320.

HAMmond, D. \& RedeKopp, L. 1997 Global dynamics of symmetric and asymmetric wakes. J. Fluid Mech. 331, 231-260.

Hannemann, K. \& Oertel JR, H. 1989 Numerical simulation of the absolutely and convectively unstable wake. J. Fluid Mech. 199, 55-88.

Huerre, P. \& Monkewitz, P. 1990 Local and global instabilities in spatially developing flows. Annu. Rev. Fluid Mech. 22, 473-537.

Huerre, P. \& Rossi, M. 1998 Hydrodynamic Instabilities in open flows. In Hydrodynamics and Nonlinear Instabilities (ed. C. Godrèche \& P. Manneville), pp. 81-294. Cambridge University Press.

Jin, G. \& BraZA, M. 1993 A nonreflecting outlet boundary condition for incompressible unsteady Navier-Stokes calculations. J. Comput. Phys. 107, 239-253.

Karniadakis, G. \& Triantafyllou, G. 1989 Frequency selection and asymptotic states in laminar wakes. J. Fluid Mech. 199, 441-469.

KocH, W. 1985 Local instability characteristics and frequency determination on self-excited wake flows. J. Sound Vib. 99, 53-83.

Le Dizès, S., Huerre, P., Chomaz, J.-M. \& Monkewitz, P. 1996 Linear global modes in spatially developing media. Phil. Trans. R. Soc. Lond. A 354, 169-212.

Leweke, T. \& Provansal, M. 1995 The flow behind rings: bluff body wakes without end effects. J. Fluid Mech. 288, 265-310.

Monkewitz, P. 1988 The absolute and convective nature of instability in two-dimensional wakes at low Reynolds numbers. Phys. Fluids 31, 999-1006.

Monkewitz, P., Huerre, P. \& Chomaz, J.-M. 1993 Global linear stability analysis of weakly non-parallel shear flows. J. Fluid Mech. 251, 1-20.

Monkewitz, P. \& NGuYen, L. 1987 Absolute instability in the near-wake of two-dimensional bluff bodies. J. Fluids Struct. 1, 165-184.

NORBERG, C. 1994 An experimental investigation of the flow around a circular cylinder: influence of aspect ratio. J. Fluid Mech. 258, 287-316.

Pier, B. \& Huerre, P. 1996 Fully nonlinear global modes in spatially developing media. Physica D 97, 206-222. 
Pier, B. \& Huerre, P. $2001 a$ Nonlinear self-sustained structures and fronts in spatially developing wake flows. J. Fluid Mech. 435, 145-174.

Pier, B. \& Huerre, P. $2001 b$ Nonlinear synchronization in open flows. J. Fluids Struct. 15, 471-480.

Pier, B., Huerre, P. \& Chomaz, J.-M. 2001 Bifurcation to fully nonlinear synchronized structures in slowly varying media. Physica D 148, 49-96.

Pier, B., Huerre, P., Chomaz, J.-M. \& Couairon, A. 1998 Steep nonlinear global modes in spatially developing media. Phys. Fluids 10, 2433-2435.

Provansal, M., Mathis, C. \& Boyer, L. 1987 Bénard-von Kármán instability: transient and forced regimes. J. Fluid Mech. 182, 1-22.

Strouhal, V. 1878 Über eine besondere Art der Tonerregung. Ann. d. Phys. u. Chem. N. F. (Leipzig) 5(10), 216-251.

Triantafyllou, G., Triantafyllou, M. \& Chryssostomidis, C. 1986 On the formation of vortex streets behind stationary cylinders. J. Fluid Mech. 170, 461-477.

Williamson, C. 1988 Defining a universal and continuous Strouhal-Reynolds number relationship for the laminar vortex shedding of a circular cylinder. Phys. Fluids 31, 2742-2744.

Williamson, C. 1996 Vortex dynamics in the cylinder wake. Annu. Rev. Fluid Mech. 28, 477-539. 